\title{
Evaluation of Portable X-ray Fluorescence Instrumentation for in situ Measurements of Lead on Contaminated Land
}

\author{
Ariadni Argyraki ${ }^{a}$, Michael H. Ramsey ${ }^{a}$ and Philip J. Potts ${ }^{b}$ \\ a Department of Geology, Imperial College, London, UK SW7 2BP \\ ${ }^{b}$ Department of Earth Science, The Open University, Walton Hall, Milton Keynes, UK MK7 6AA
}

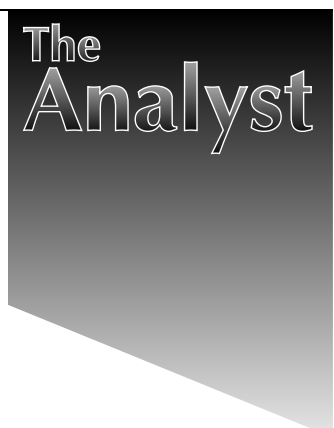

The performance of the Spectrace TN 9000 portable $\mathrm{X}$-ray fluorescence (P-XRF) instrument for in situ sampling and analysis of contaminated soil was evaluated. The method was compared with laboratory analysis of the samples using ICP-AES and XRF as established methods for the assessment of contaminated land. The trueness of the field-based P-XRF results was affected by the soil moisture and the surface roughness of the in situ samples, after the correction of which, no bias was observed between the analytical results of the comparative methods. Relatively large measurement uncertainty $( \pm 55 \%$ for $\mathbf{P b})$ was caused by the small sample mass analysed and the small scale heterogeneity of the sample. This uncertainty was quantified using duplicate measurements and does not impair the delineation of 'hot spots' of contamination as it contributes less than $20 \%$ to the total variance. General advantages and limitations of the P-XRF methodology for the investigation of contaminated land were assessed and suggestions are made for the optimisation of the methodology.

Keywords: Portable X-ray fluorescence instrumentation; in situ measurement; lead; contaminated land

In situ measurements of soils at contaminated sites potentially have the advantages of giving both a rapid assessment of the concentration of the contaminant and also first hand information on its spatial distribution and the degree of heterogeneity in an undisturbed position. The disadvantage of in situ measurement is often the high degree of uncertainty associated with the measurement, caused by factors such as the heterogeneity. If, however, this uncertainty can be quantified realistically, then these measurements can be fit for many purposes of environmental interpretation. Conventional schemes of analysis can usually be divided into two basic stages: (i) the collection of samples in the field, and (ii) the preparation and analysis of these samples in the laboratory, using a variety of analytical methods. The selection of the most appropriate sample preparation and analysis method depends on the sampling medium, the scope of the investigation and the availability of facilities. One well established procedure for the analysis of contaminated soils is to collect samples of top soil (e.g., $0-150$ $\mathrm{mm}$ ) using a hand auger, sieve and collect the $<2 \mathrm{~mm}$ fraction, grind to a fine powder and then use an acid extraction procedure, followed by ICP-AES to determine selected analytes. ${ }^{1}$ However, it is important to appreciate that the estimated values of contaminant concentration from such investigations may vary due to sampling errors associated with the particular sampling design used in the field. ${ }^{2}$

$\mathrm{X}$-ray fluorescence (XRF) is also a well established methodology for the assessment of contaminated land. Samples may be dried and then analysed as loose powders or alternatively prepared as compressed powder pellets. Ease of sample preparation as well as analytical trueness and high precision are advantages of this methodology. ${ }^{3}$
Field-based portable XRF (P-XRF) instrumentation offers potential advantages over other laboratory techniques. By undertaking analyses of contaminated soil in situ, and thereby avoiding the necessity of removing samples, P-XRF has the potential of giving both a rapid assessment of the concentration of the contaminant and also immediate information on its spatial distribution and degree of heterogeneity without disturbing the location.

One disadvantage of in situ measurements is the high degree of uncertainty that may be associated with the measurement, caused by sample heterogeneity effects. If, however, this uncertainty can be quantified realistically, then these measurements may be fit for many purposes of environmental interpretation. As noted for in situ measurements in general, the potential problem of high measurement uncertainty can be addressed by its realistic quantification and recognition in environmental interpretation.

Field-based P-XRF instruments are becoming increasingly important in assessments of contaminated land. Their performance for the investigation of contaminated land has recently been evaluated by several workers but after removal of the soils to a field laboratory. Specifically, P-XRF devices have been used for the immediate delineation of $\mathrm{Cr}$ source contamination 'hot spots' 4 and the spatial distribution of $\mathrm{Pb}$ concentration in residential soils. ${ }^{5}$ P-XRF methodology has also been used to provide data for remedial activities at sites contaminated with $\mathrm{Pb}$ and $\mathrm{As}^{6}$ and the determination of $\mathrm{Pb}$ in urban soil and dust samples. ${ }^{7}$

Comparisons of the estimates of elemental concentrations made using P-XRF with estimates from other analytical methods such as ICP-AES and AAS have revealed data of acceptable quality when the portable methodology is used properly. However, a slight bias between the XRF results and those of the other methods has been reported. ${ }^{4-9}$ A significant problem of the P-XRF methodology, addressed in most of the above studies, is the use of appropriate calibration samples.

This paper uses in situ rather than laboratory-based measurements using a Spectrace TN 9000 P-XRF instrument for the determination of $\mathrm{Pb}$ in soil. The main aim of this work was to evaluate the performance of a field-based P-XRF instrument for the determination of $\mathrm{Pb}$ in contaminated soil in comparison with an established method of analysis, i.e., hand augering followed by laboratory-based ICP-AES. To facilitate the evaluation, the field chosen for this assessment had previously been studied in some detail as part of an on-going study of environmental sampling procedures. ${ }^{2,10,11}$ The use of P-XRF for spatial mapping, for locating contamination 'hot-spots' and for flexibility in the design of sampling protocols was also assessed. Results are used to demonstrate both the advantages and limitations of the technique. The rational optimisation of the sampling and analytical protocol using P-XRF for the investigation of contaminated land, both in situ and in the laboratory, was another objective of this work. The last objective was to identify needs for further development work, which will enable the ultimate limits of performance of P-XRF to be approached. 


\section{Experimental \\ $P$-XRF}

Field measurements were undertaken with a Tracor Northern Spectrace TN 9000 P-XRF instrument on hire from Thermo Unicam (Cambridge, UK). The instrument consisted of a handheld analyser unit and a portable spectrum acquisition and data processing unit (Fig. 1). The analyser unit incorporated three radioactive isotope excitation sources: ${ }^{55} \mathrm{Fe},{ }^{109} \mathrm{Cd}$ and ${ }^{241} \mathrm{Am}$ and a mercury(II) iodide X-ray detector. To undertake an analysis in the field, the analyser unit was placed against the sample surface which was excited with each of the three sources in turn. For the analysis of samples in the laboratory (e.g., powdered soil), the analyser unit was mounted in a laboratory stand with the analyser window pointing vertically and covered by a sample lid, the position of which (open or closed) was interlocked to the operation of the instrument. Samples were then conveniently contained in conventional XRF sample cups, placed in position over the window, and analysed once the lid was closed. Whatever the form of sample presentation, spectra were accumulated in the spectrum acquisition and data processing unit and at the end of the preset count time, fluorescence intensities were measured using a spectrum deconvolution procedure based on region-of-interest integration. Data were then corrected for matrix effects and quantified using a fundamental parameter procedure. Quantitative results were displayed for immediate appraisal and stored for subsequent downloading to an external microcomputer via an RS232 interface.

The performance of this XRF technique in the laboratory has been described by Potts et al. ${ }^{3}$ These earlier studies were undertaken on a range of silicate rock reference materials prepared as compressed powder pellet samples, and were designed to demonstrate the basic performance characteristics of the technique. Using a count time (live time) of $200 \mathrm{~s}$ per source, repeatability precision was found to be in the range $0.45-1.8 \%$ for the major elements and $2-5 \%$ for trace elements. A high degree of linearity was also achieved in the relationship between analysed and expected values for the 70 international reference materials investigated. The detection limit for $\mathrm{Pb}$ was found to be $39 \mu \mathrm{g} \mathrm{g}^{-1}$, representative of a $200 \mathrm{~s}$ count time.

\section{ICP-AES}

Field-based P-XRF results were compared with an established ICP-AES technique. Samples representing the top 0-150 mm surface of the soil were removed with a hand auger and returned to the laboratory for analysis. There, samples were dried, ground in a pestle and mortar and analysed by ICP-AES after

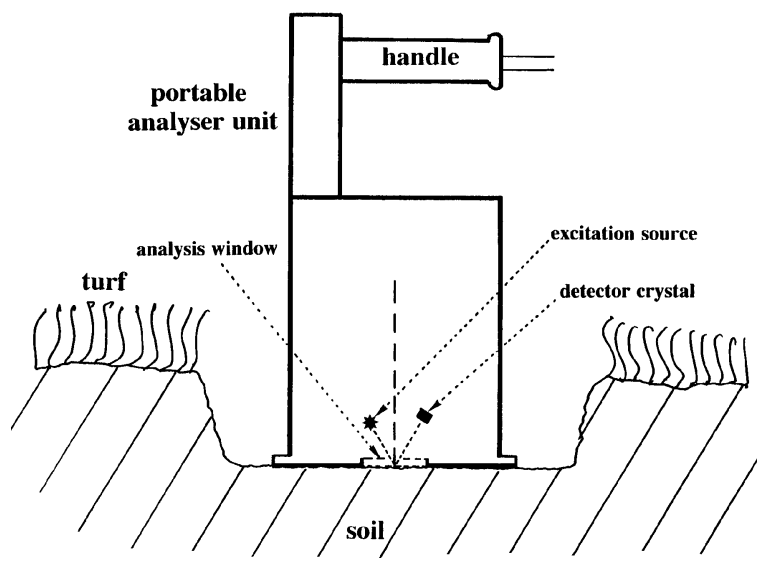

Fig. 1 Schematic representation of P-XRF instrumentation for in situ soil analysis. acid extraction. Full details of the procedure can be found elsewhere. ${ }^{1}$

\section{The Site}

The site selected for study was a fallow field at Bolehill near Wirksworth, Derbyshire, UK. This site was used in Medieval times for $\mathrm{Pb}$ smelting. Recent interest arose, therefore, in the possibility of using the presence of high levels of $\mathrm{Pb}$ contamination known to be present in the soil to model the mobility of this contaminant in the environment. ${ }^{12}$ Previous investigations by auger sampling and ICP-AES analysis had revealed mean $\mathrm{Pb}$ concentrations of $6229 \mu \mathrm{g} \mathrm{g}^{-1}$ in the top 0-150 mm soil layer over the site. ${ }^{2}$ The spatial distribution of the element was also well known from both this pilot study (Fig. 2), and also a proficiency testing and collaborative sampling trial which had been based on part of the same field. ${ }^{10,11}$ Useful information about the sampling variance was available from these trials as well as analytical bias in the contributed data based on the analysis of six CRMs and SRMs (BCR CRM 141, BCR CRM 142, BCR CRM 143, NIST SRM 2709, NIST SRM 2710 and NIST SRM 2711).

\section{Sampling Protocol}

On the basis of these previous site investigations, a field of approximately $10000 \mathrm{~m}^{2}$ in area was selected for study. This area was known to include an area containing the highest concentration of contaminants (contamination 'hot spot') as well as lower concentrations and was, therefore, judged to be both suitable for evaluating the performance of P-XRF against ICP-AES as well as testing the capabilities of P-XRF for 'hot spot' location. The field was first marked out with a $30 \times 30 \mathrm{~m}$ grid using a $30 \mathrm{~m}$ triangulation tape (Fig. 3). Three sets of measurements were then undertaken. (i) An orienteering survey at the nodes of the $30 \mathrm{~m}$ grid. (ii) On the basis of these measurements a second, more dense $10 \times 10 \mathrm{~m}$ grid was investigated on the same day, focusing on two areas identified as potential 'hot spots' of contamination on the basis of the first set of measurements. (iii) Finally, measurements were taken on two $1 \mathrm{~m}^{2}$ areas to characterise the small scale lateral variation of the metals in soil and to elucidate further the problems of sample heterogeneity. These last two areas were selected at both a 'hot spot' and a lower concentration area at the field.

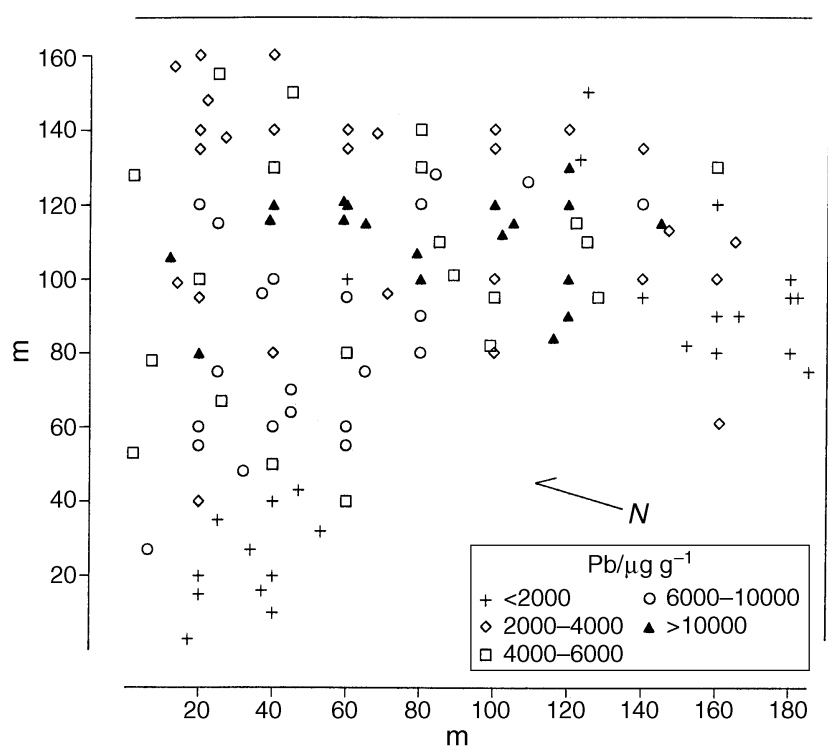

Fig. 2 Sample locations and $\mathrm{Pb}$ concentration in soil from previous study of the contaminated field in Derbyshire. 
At each location selected for analysis, turf was removed to a depth of about $30 \mathrm{~mm}$ over an area of approximately $150 \times 150$ $\mathrm{mm}$ using a spade. The removal of the turf was necessary to expose unvegetated topsoil and to achieve a reasonably flat surface for P-XRF analysis, keeping in mind that the XRF signal for $\mathrm{Pb}$ originates from the top $1.5 \mathrm{~mm}$ surface layer. Duplicate measurements were taken at each sampling point at a distance of $2 \mathrm{~m}$ from the initial sampling location, so that an estimate could be made of measurement precision (including sampling error). This distance was selected to represent the location error of the sampling locations on the field given the surveying technology employed (30 $\mathrm{m}$ tape, triangulated). Auger samples $0-150 \mathrm{~mm}$ deep and $25 \mathrm{~mm}$ in diameter were also taken in each of the measuring sites for laboratory analysis by XRF and ICP-AES.

\section{Analysis Protocol}

Relatively short count times were selected for the P-XRF instrument to maximise the rate at which sites could be measured rather than to optimise detection limits. Selected count times were, therefore, $50 \mathrm{~s}$ for the ${ }^{109} \mathrm{Cd}$ source, $20 \mathrm{~s}$ for ${ }^{55} \mathrm{Fe}$ and $5 \mathrm{~s}$ for ${ }^{241} \mathrm{Am}$. The relatively high detection limit for $\mathrm{Pb}$ obtained under these conditions (calculated to be about $39 \mu \mathrm{g}$ $\mathrm{g}^{-1}$ ) did not affect the results significantly because of the high concentration of the contaminant in the soil at this site. The time required for duplicate P-XRF measurements at each of the sampling locations was approximately 3-5 min, allowing 24 sampling locations to be covered in half a working day.

The samples collected for laboratory analysis were dried at $65^{\circ} \mathrm{C}$ (to prevent excessive 'caking' of soil that results if $100^{\circ} \mathrm{C}$ is used), disaggregated in a pestle and mortar and the $<2 \mathrm{~mm}$ fraction was ground (to $<100 \mu \mathrm{m}$ ) using an agate ring grinder. Analytical test portions of these samples were analysed as loose powders by laboratory-based P-XRF, using a sample cup fitted with a $6 \mu \mathrm{m}$ thick polyester film. The measurements were taken for 100,40 and $20 \mathrm{~s}$ using ${ }^{109} \mathrm{Cd},{ }^{55} \mathrm{Fe}$ and ${ }^{241} \mathrm{Am}$ radioisotope sources, respectively, simulating the conditions in a field laboratory and improving on the measuring times used in situ. The same samples were analysed using ICP-AES after treatment with a mixture of nitric and perchloric acids to solubilize the analytes, which were presented for analysis in dilute hydrochloric acid solutions. ${ }^{1}$

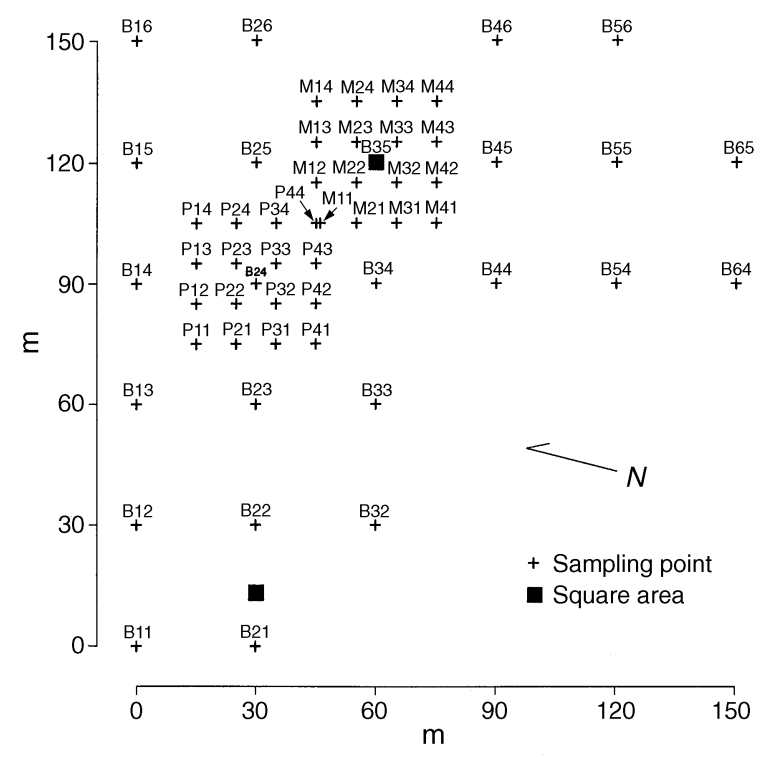

Fig. 3 Sample locations for the in situ P-XRF measurements and collection of auger samples, showing the three sets of measurements: the initial $30 \mathrm{~m}$ grid, the $10 \times 10 \mathrm{~m}$ grid and the two $1 \mathrm{~m}^{2}$ areas.
For the analytical quality control, four reference materials (HRM 1, HRM 2, NIST SRM 2710 and HRM 31) in the form of compressed pellets were run periodically between the measurements in the field. In the laboratory-based XRF analysis, five reference materials were used (HRM 1, HRM 2, HRM 31, GXR-2 and NIST SRM 2710) and for the ICP-AES analysis seven reference materials (BCR CRM 141, BCR CRM 142, BCR CRM 143, NIST SRM 2709, NIST SRM 2710, NIST SRM 2711 and HRM 31) were employed. HRMs are house reference materials prepared at Imperial College for measuring the analytical bias. They are less expensive than the CRMs and their accepted value has been determined against existing CRMs. In particular, HRM 31 has a matrix composition that matches the sample composition of this particular sampling target. It was prepared using a composite material taken previously from the same field. The concentrations of both the analytes and the matrix elements in this HRM were in very close agreement with those of the samples. Duplicate analytical measurements were undertaken on each of the sampling duplicates in the laboratory, for ten of the sampling locations, in order to provide estimates of both the sampling and analytical precision.

\section{Results}

\section{Measurement and Sampling Variance}

The first evaluation of performance was to estimate the proportion of the total variance of elemental concentration in the three data sets that could be apportioned to sampling and analysis. $\mathrm{Pb}$ data from the three sets of measurements (field measurements by P-XRF, laboratory measurements by P-XRF and laboratory measurements by ICP-AES) were evaluated by an analysis of variance (ANOVA) routine and judged against criteria proposed by Ramsey et al. ${ }^{13}$ These criteria, developed to ensure the appropriate interpretation particularly of environmental data, propose that the sampling variance plus analytical variance (together called the measurement variance) should not exceed $20 \%$ of the total variance of a data set. The balance, 'geochemical' variance, contains information about the geochemical or distribution behaviour of an analyte which can only be interpreted with confidence if this measurement variance threshold is not exceeded. Accordingly, the proportion of the total variance of $\mathrm{Pb}$ measurements contributed by the processes of sampling and analysis for all three techniques is shown in Fig. 4. The proposed limit is not exceeded for any of the sets of data. For the field-based P-XRF measurements, it was not possible to separate the analytical and sampling variance components of the measurement variance [Fig. 4(a)], but it is clear that sampling is the dominant contributor for both laboratory-based P-XRF and ICP-AES [Fig. 4(b) and (c)]. The similarity between the percentage of measurement variance for both the field- and laboratory-based P-XRF also suggests that this conclusion would be true for the field-based P-XRF measurements. The uncertainty on the measurements of the field-based P-XRF was also calculated as $200 S_{\text {meas }} / \bar{x}$, where $S_{\text {meas }}$ is the estimated measurement variance by robust ANOVA and $\bar{x}$ the robust mean of the duplicate measurements. The value of this uncertainty was $\pm 55 \%$ of the mean value.

\section{Bias}

Bias was evaluated from NIST SRM 2710, which was analysed for all contributing measurement techniques. This reference material was selected because the matrix and analyte concentrations match approximately the samples under consideration. Results for $\mathrm{Pb}$ are listed in Table 1. For $\mathrm{Pb}$, the bias of $-5.5 \%$ for ICP-AES probably originates in under-correction of matrix effects. For P-XRF, the bias of $-11 \%$ for both field and laboratory measurements may reflect a mis-match between the 
composition and degree of compaction of this reference material and that of a 'typical' soil used to determine the fundamental parameter coefficients required for the matrix
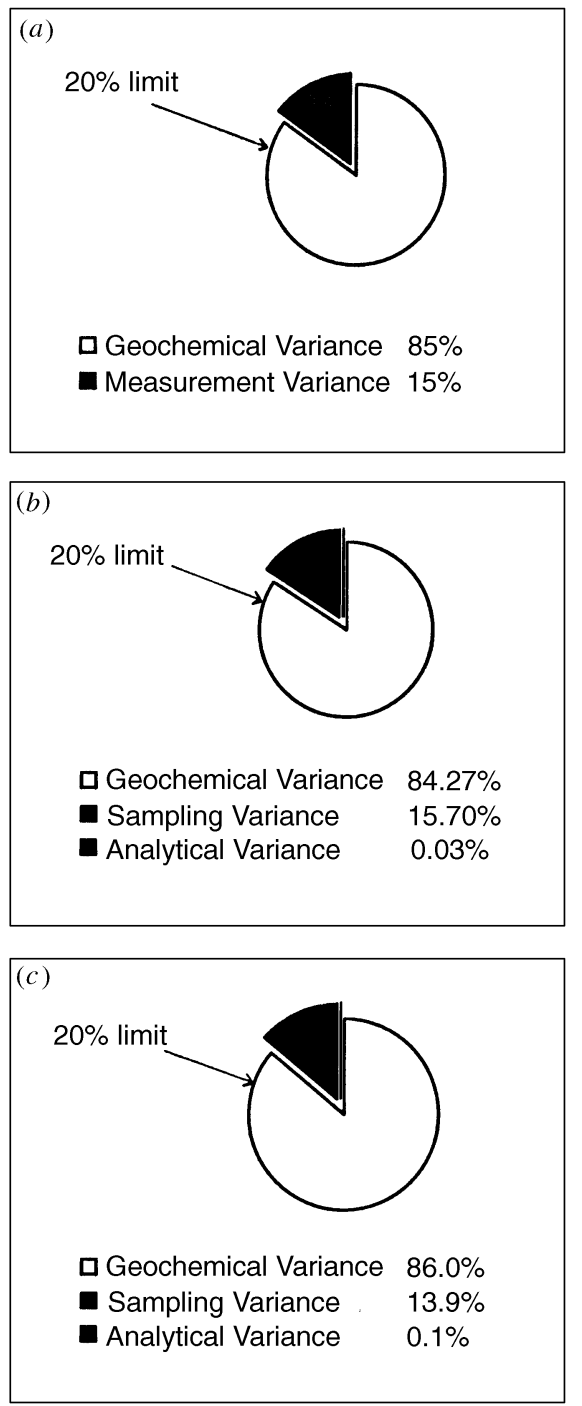

Fig. 4 ANOVA pie charts for $\mathrm{Pb}$ in soil, showing the proportions of sampling, analytical and geochemical variances for all three analytical techniques. (a) P-XRF, field data; (b) P-XRF, laboratory data; (c) ICP-AES data. The $20 \%$ limit for the measurement variance is not exceeded for any of the analytical methods.

Table 1 Comparison of results for the analysis of NIST SRM 2710 by the three methods, showing acceptable levels of analytical bias. Bias for ICPAES probably originates in under-correction of matrix effects. For P-XRF the bias may reflect a mis-match between the composition of the reference material and that of the soil used in the matrix correction procedure ( $n$ : number of analyses)

\section{NIST SRM 2710}

$\mathrm{Pb}$

Accepted value/ $\mathrm{ug} \mathrm{g}^{-1}$

5532

Measured by field-based P-XRF/ $/ \mathrm{g} \mathrm{g}^{-1}$

4935 $(n=10)$

Bias (\%)

Measured by laboratory-based P-XRF/ $\mu \mathrm{g} \mathrm{g}^{-1}$

$-10.8$

$(n=4)$

Bias $(\%)$

Measured by ICP-AES/ $\mu \mathrm{g} \mathrm{g}^{-1}$

4898

$(n=2)$

Bias $(\%)$

$-11.5$

5228

$-5.5$ correction procedure. The fact that the reference material used was pelletized, but the samples analysed in laboratory-based PXRF were not, could have been a potential source of bias. However, when powdered samples were analysed by both laboratory-based P-XRF and ICP-AES no significant bias was detected.

When evaluating these data sets, it must be stated that there are some limitations in the use of a dry, finely ground, pelleted reference material to estimate the bias of field measurements by P-XRF made on moist, uncompacted and unground soils with a flat but irregular surface. Nominally, however, these levels of bias are probably acceptable and 'fit-for-purpose' in the context of this work, but further investigations of the origin of this bias are presented below.

Bias can also be estimated by comparing the three sets of measurements against each other (i.e., relative bias). A direct comparison by simple linear regression of the measurements for $\mathrm{Pb}$ by field-based P-XRF against those by ICP-AES show a rotational 'bias' of $-57 \pm 6 \%(1 s)$ in the former (Fig. 5). However, this 'bias' includes a measure of sampling bias as well as purely analytical bias, because the form and nature of the samples used in the comparison were not identical. The fieldbased P-XRF measurements are representative of the soil surface composition of $\mathrm{Pb}$ to a maximum depth of about 1.5 $\mathrm{mm}$, whereas the ICP-AES results represent the homogenised 0-150 $\mathrm{mm}$ auger sample. Furthermore, the field-based P-XRF measurements were made on wet, uncompacted soils, whereas the ICP-AES measurements were made on dried, sieved and homogenised powders. A bias between the two techniques is to be expected, therefore, but to understand the capabilities and limitations of the P-XRF technique, it is relevant to investigate the components of this bias.

When the bias for $\mathrm{Pb}$ between field-based $\mathrm{P}-\mathrm{XRF}$ and laboratory-based P-XRF is compared, a similar rotational 'bias' is apparent, namely $-52 \pm 7 \%(1 \mathrm{~s})$, compared with $57 \pm 6 \%$ for the field-based P-XRF versus laboratory-based ICP comparison. This suggests that the bias is not produced by a difference in the performance of the analytical technique, which is identical here, but is due to one of these other factors of sampling and sample preparation. The use of simple least squares linear regression has been shown to have limitations in the detection of bias between analytical methods, ${ }^{14}$ but such limitations are considered not to have affected this broad conclusion.

\section{Origins of Bias}

One hypothesis for the apparent low 'bias' of the field-based P$\mathrm{XRF}$ measurements is that the $\mathrm{Pb}$ content of the top few millimetres of the soil is systematically lower than that in the

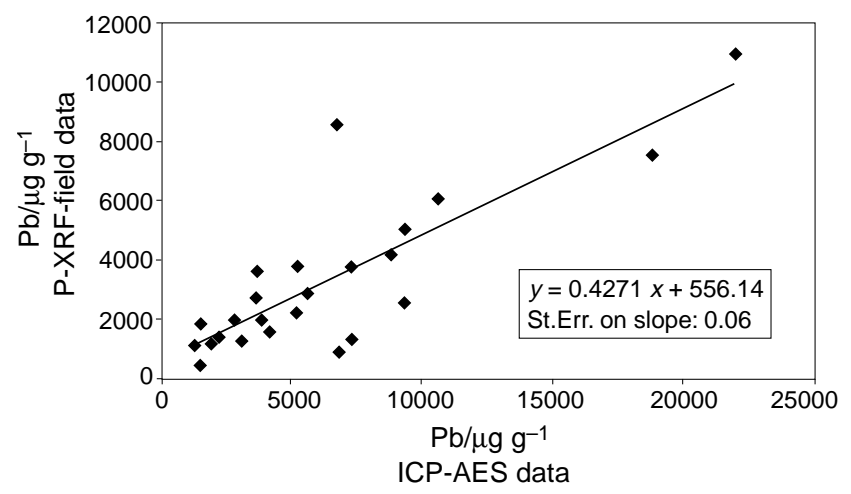

Fig. 5 Simple linear regression of field-based $\mathrm{P}-\mathrm{XRF} \mathrm{Pb}$ measurements against ICP-AES Pb measurements showing a rotational bias of $-57 \pm 6 \%$ between the two analytical methods. 
$150 \mathrm{~mm}$ sample taken for both laboratory-based P-XRF and ICP-AES analysis. To test this hypothesis, a $62 \mathrm{~mm}$ core of topsoil was taken in an area of the field with high $\mathrm{Pb}$ concentration, and progressively cut to remove 11 slices of around $6 \mathrm{~mm}$ thickness. The core of soil was analysed in situ in the field by P-XRF before each slice was removed (Fig. 6). Each slice was also analysed as a dried powder by laboratory-based ICP-AES. The $\mathrm{Pb}$ profiles produced by this 'sliced core experiment' show little systematic spatial trend with depth of core (Fig. 7), and in particular, there is no particular depletion in the top few millimetres, as would be required to confirm the hypothesis. A second core showed a different vertical variation in $\mathrm{Pb}$ concentration, but again no significant depletion at the surface. It is concluded, therefore, that at the site investigated in this study, surface analysis by P-XRF gives results that are not biased due to vertical variation in $\mathrm{Pb}$ concentration in the soil.

Two other potential causes for the low 'bias' of the fieldbased P-XRF measurements are the moisture content of the soil and the rough surface of the soil used for excitation. These factors were corrected to assess their relative contributions to the bias. The moisture content of each slice of the core experiment was determined and ranged from $6.83 \%$ to $19.09 \%$

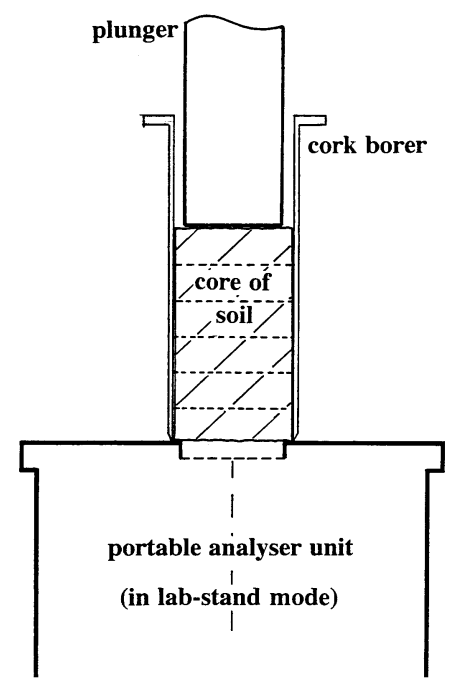

Fig. 6 Schematic representation of the 'core experiment' set-up.

$\mathrm{Pb} / \mu \mathrm{g} \mathrm{g}^{-1}$

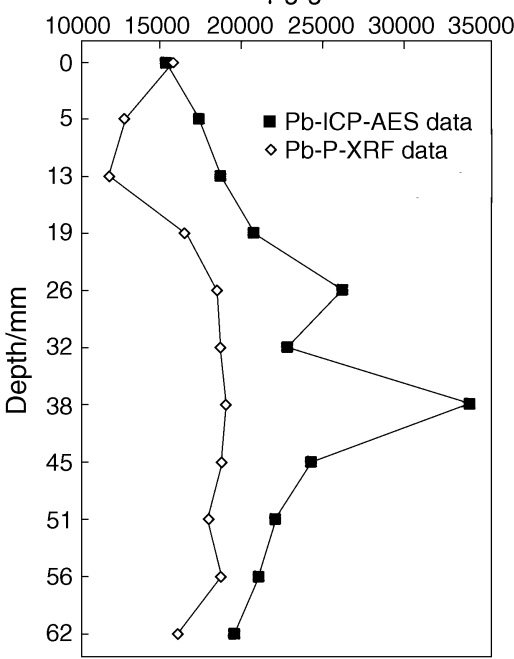

Fig. $7 \mathrm{~Pb}$ concentration in soil as determined in the core by the two analytical techniques, showing little systematic spatial trend in the core and no depletion in the top few millimetres. $\mathrm{m} / \mathrm{m}$. When a correction was made for moisture to the fieldbased P-XRF measurements, an initial bias of $-19.9 \%$ against ICP-AES determinations for this particular experiment was reduced to $-7.2 \%$ (Fig. 8). In order to compensate for the roughness of the soil surface, a Rayleigh scatter correction ${ }^{15}$ was made. After this correction, the bias was effectively eliminated, giving a non-significant bias of $+0.8 \%$ over the 19 slices of both cores.

It would appear, therefore, that the cause of the apparent 'bias' of the field-based P-XRF measurements, at least for these two cores, is entirely due to the combined effects of soil moisture and surface roughness. In practice, the Rayleigh scatter correction can be applied routinely with minimal extra measurement time, but the determination of moisture correction will need to be either a retrospective correction, or require the deployment of a field probe.

\section{Mapping}

The possibility of mapping the spatial distribution of metal contamination across a site in real time is one of the significant advantages of using field-based P-XRF. A further strength of the technique is that 'hot spots' can be investigated in more detail during the same visit at the sampling target. Measurements from the initial $30 \mathrm{~m}$ grid indicated two 'hot spots' with $\mathrm{Pb}$ concentrations of over $8000 \mu \mathrm{g} \mathrm{g}^{-1}$ (Fig. 9). To investigate these 'hot spots' further, two secondary $10 \mathrm{~m}$ grids were set up centred on the candidate 'hot spots' (Fig. 9). Interestingly, the existence of the eastern 'hot spot' was substantiated by a cluster of similarly high $\mathrm{Pb}$ concentrations. The western 'hot spot', however, was not substantiated, with most of that $10 \mathrm{~m}$ grid being lower than $6000 \mu \mathrm{g} \mathrm{g}^{-1}$. Given the high sampling variance (i.e., within-location) found at this site (15\% of total), it would be expected that occasional isolated high values will occur by chance, without being confirmed by high data from adjacent sites to substantiate a 'hot spot'. The use of field-based P-XRF allows such 'spurious hot spots' to be eliminated at the time of the survey. Any bias in the measurements would not directly affect the investigation of this spatial coherence of the 'hot spots', but only affect their intensity and extent. Fitnessfor-purpose criteria should be considered in order to determine the significance of any bias in the measurements. In this instance, the estimated bias of $-57 \%$ for $\mathrm{Pb}$ between the in situ $\mathrm{P}$-XRF measurements and those made in the laboratory does not affect the spatial delineation of the 'hot spots' since the observed metal concentrations are well above the limits for contaminated land. In general, given the uncertainty of $\pm 55 \%$ on

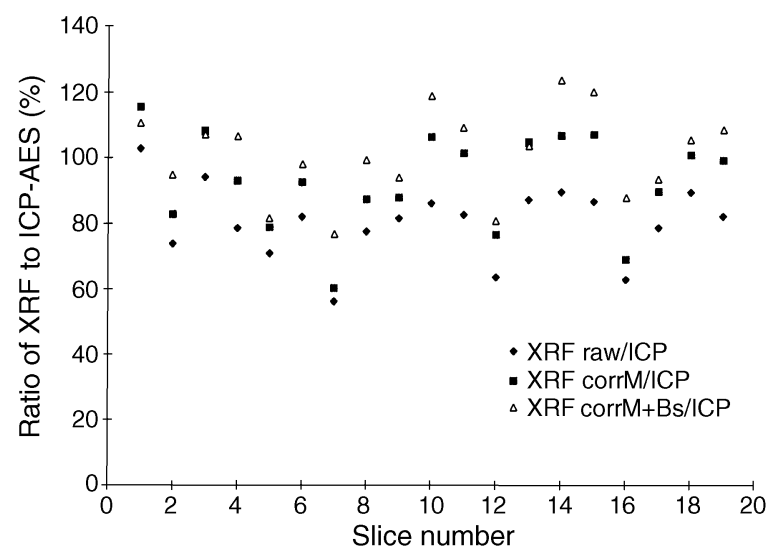

Fig. 8 Comparison data for $\mathrm{Pb}$ showing the ratio of XRF to ICP-AES analyses for the raw data, XRF data corrected for moisture and XRF data after moisture and Rayleigh scatter corrections. The bias after the corrections is effectively reduced to a non-significant level of $+0.8 \%$. 
the field-based P-XRF measurements, the $\mathrm{Pb}$ concentration would have to be over $4000 \mu \mathrm{g} \mathrm{g}^{-1}$ in order to be recognised as a 'hot spot' against the background of $2000 \mu \mathrm{g} \mathrm{g}-1$.

Spatial mapping on the smallest scale (i.e., <1 m) using field-based P-XRF was used to investigate the heterogeneity of $\mathrm{Pb}$ on what is called the 'within-location' scale, in the larger scale surveys. The square metre surrounding the eastern 'hot spot' was surveyed using 16 samples, four taken at each of the distances from the centre of 500, 250, 120 and $50 \mathrm{~mm}$ (Fig. 10). The pattern of $\mathrm{Pb}$ concentrations measured does not conform to a single smooth maximum. Although high concentrations ( $>14000 \mu \mathrm{g} \mathrm{g}^{-1} \mathrm{~Pb}$ ) dominate the central area, there is also a similar high value at the eastern edge, and also a low value $\left(8810 \mu \mathrm{g} \mathrm{g}^{-1}\right)$ close to the centre. If the variance of the $\mathrm{Pb}$

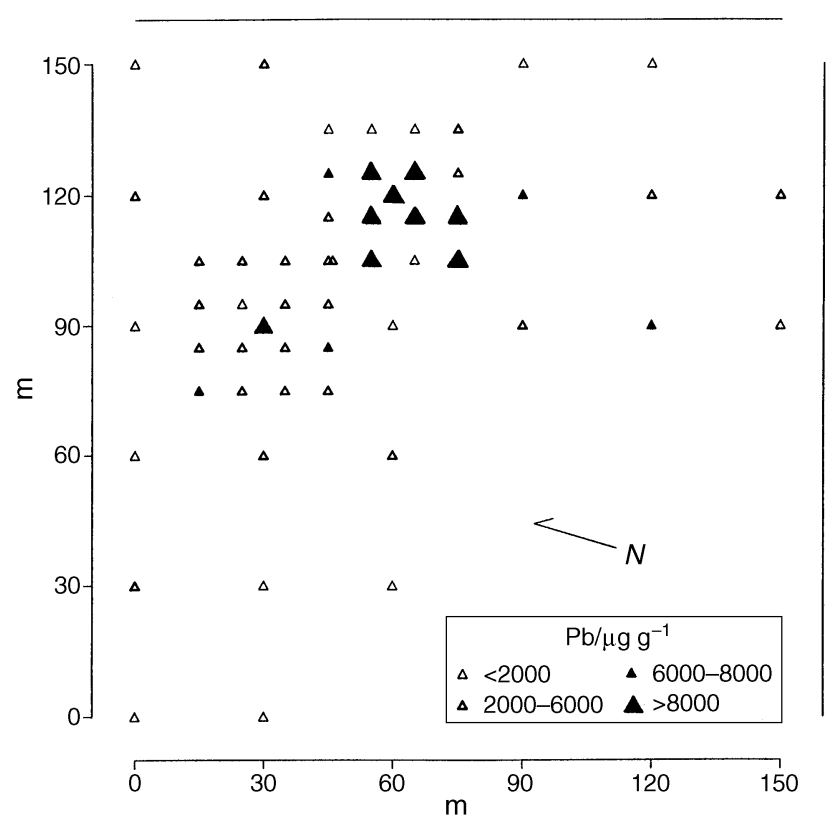

Fig. 9 Spatial mapping of $\mathrm{Pb}$ concentration determined by $\mathrm{P}-\mathrm{XRF}$, showing the iterative sampling design with initial $30 \mathrm{~m}$ grid followed by the $10 \mathrm{~m}$ grid centred on candidate 'hot spots' $(\mathrm{Pb}>8000 \mu \mathrm{g} \mathrm{g}-1)$. The eastern 'hot spot' is confirmed by a cluster of high values, whereas the western 'hot spot' is not confirmed.

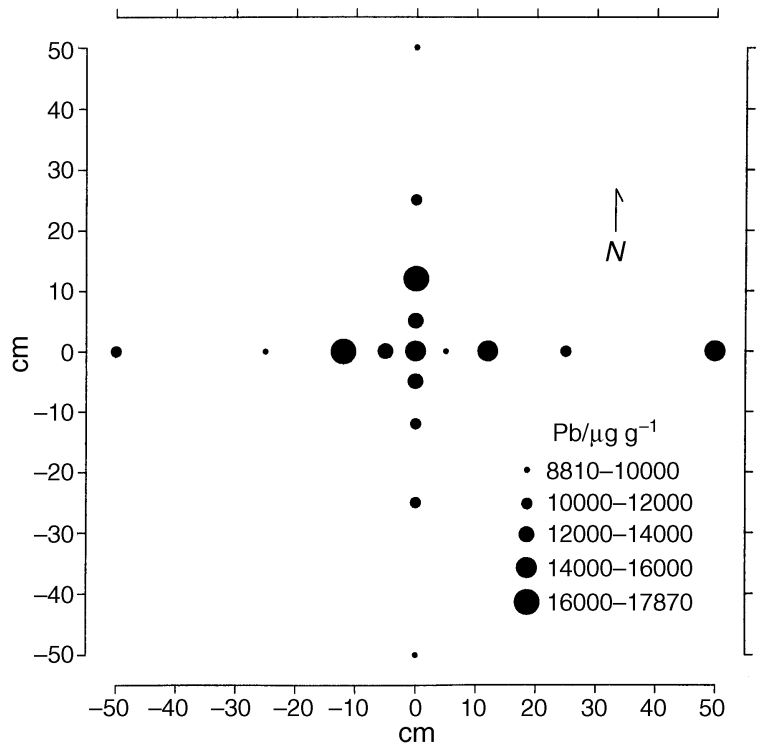

Fig. 10 Concentration of $\mathrm{Pb}$ in soil in the $1 \mathrm{~m}^{2}$ area over the contamination 'hot spot' on the eastern side of the field, showing no systematic trend in the highest values. concentration is estimated from the four replicates at each distance, then there is no systematic change with distance $(\mathrm{RSD}=28,24,32$ and $18 \%$ for the four distances mentioned above). Furthermore, this variance within $1 \mathrm{~m}$ is not significantly different from that measured at the 10 or $30 \mathrm{~m}$ scales, when expressed in units relative to the mean value at the corresponding scale. Similar results were extracted from the second $1 \mathrm{~m}^{2}$ site located in the area with the lowest metal concentrations.

\section{Discussion}

The potential of P-XRF for the in situ investigation of metal contaminated land has been evaluated, showing the strengths and disadvantages of the methodology. The possibility of immediate estimates of metal contamination of soil by in situ measurement is the most important advantage of the method over previous studies which have used P-XRF with the soil removed to an on-site laboratory. Large amounts of data can be obtained in a timely and cost-efficient manner during a single visit to the sampling target.

Detection limits were sufficiently low to measure elevated levels of contamination with sufficient precision for several metals of interest (e.g., $\mathrm{Pb}, \mathrm{Zn}$ and potentially $\mathrm{Cu}, \mathrm{Ni}, \mathrm{As}, \mathrm{Ba}$ ) in the particular field under study. The fact that the contaminants were present in high concentrations enhanced the abilities of the method but, since the P-XRF detection limits are well below the regulatory limits for most metals on contaminated land, the methodology is considered to be very useful for such investigations.

The rapid analysis time for a suite of several metals (e.g., 2 $\min$ ) is another advantage. However, the measurement time may need to be extended for sampling targets where elemental concentrations are lower than in this study. Because of its ability for rapid analysis, P-XRF methodology facilitates the use of iterative sampling designs. For example, a follow-up survey can be implemented to investigate any apparent 'hot spots', during a single site visit.

Satisfactory analytical quality was observed in the in situ analysis, giving good analytical precision (e.g., 1\%). However, the overall measurement precision is determined by the degree of heterogeneity of the sample site, effectively increasing the uncertainty of the analytical measurements. Another important advantage of the P-XRF methodology is the potential for the study of the heterogeneity of the sampling target at any relevant scale. In fact, P-XRF provides a tool for the investigation of in situ heterogeneity of the metal concentration across the sampling target, down to the centimetre scale.

Disadvantages of the method include the very small size of sample from which the XRF signal originates $\left(\approx 1 \mathrm{~cm}^{3}\right)$ which, although useful for mapping the heterogeneity, may give results that are not representative of the surface composition to within an acceptable measurement precision. However, at the site studied here, although the measurement variance by field-based $\mathrm{P}-\mathrm{XRF}$ was high, it was still a sufficiently small fraction of the total variance to be acceptable for the purposes of this study. If the small depth of an analysis $(1.5 \mathrm{~mm})$ is not considered representative, there is the possibility of averaging measurements over a greater depth, as in the sliced core study described above, or indeed extending measurements over even greater depths by the analysis of drill cores.

Bias in the estimates of $\mathrm{Pb}$ concentrations by field-based $\mathrm{P}$ XRF was quantified by comparison of the measurements with those from the laboratory analysis of the corresponding samples using ICP-AES and P-XRF. The problem of apparent bias was overcome in this instance by making corrections for soil moisture and surface roughness of the soil surface analysed. In general, recognition of the sources of bias is important for the evaluation and improvement of the P-XRF methodology. 
Detection limits are not sufficiently low to quantify some elements (e.g., Cd) at typical background concentrations in soils (e.g., 0.1-0.5 $\left.\mu \mathrm{g} \mathrm{g}^{-1} 16\right)$. Also, a slightly longer survey time was required on the site compared with that required for the simple taking of soil samples. The method gains credit, however, in the total time spent for analysis, since the time for sample preparation is minimised.

Finally, disadvantages related to the logistics of the use of the method include the relatively high capital cost of equipment for occasional use. Also, the time and expense required for compliance with licensing and safety regulations necessary for the use of instrumentation containing radioactive excitation sources is a potential problem that will vary between different countries.

When laboratory-based P-XRF is used with larger, dried and homogenised samples, many of these disadvantages disappear, but with the simultaneous loss of one of the main advantages of the technique: that of immediate measurement output and information on in situ variations in concentration whilst on the sampling target.

\section{Conclusions}

The performance of P-XRF using Spectrace TN 9000 instrumentation as a field sampling and analytical technique has been evaluated for the in situ assessment of contaminated land. The technique has proved to be a useful and fit-for-purpose, powerful tool, capable of giving precise and very rapid analytical results for the determination of $\mathrm{Pb}$ concentrations in soil.

The method was compared with both ICP-AES analysis of solutions derived from the soil samples and laboratory analysis of powdered samples using XRF, as established methods for the assessment of contaminated land. A rotational bias of $-57 \%$ was calculated between the in situ field-based P-XRF and the ICP-AES measurements for $\mathrm{Pb}$. The magnitude of bias between the field-based P-XRF measurements and the laboratory-based $\mathrm{P}-\mathrm{XRF}$ results was estimated to be $-52 \%$ for the same element. The origin of this bias was shown to be due largely to factors related to the nature of the sample. Specifically, the soil moisture and the surface roughness of the samples are the two factors which, without correction, affected the trueness of the results. When a correction was applied for these factors at two locations within the field, the bias for $\mathrm{Pb}$ measurements was effectively reduced to a statistically insignificant level.

Field-based P-XRF measurements were capable of rapidly locating contamination 'hot spots' in the field. Furthermore, the detected contamination 'hot spots' on the initial $30 \mathrm{~m}$ grid could be investigated during the same working day by setting up a more dense sampling grid around the areas with the highest metal concentrations. Bias in uncorrected field-based P-XRF results did not affect the spatial coherence of the 'hot spots' but only their intensity and extent. Consequently, the effect of the bias, if uncorrected, would be vital in evaluating the extent of metal contamination if concentrations were close to a regulatory limit.

An important characteristic of the field-based P-XRF methodology is the ability to investigate the small scale variability of metal content in soil. This is particularly useful in sampling targets showing a high degree of heterogeneity with respect to the metal distributions. The degree of heterogeneity can easily be quantified by estimating the variability in different spatial scales within the field.

The limitations of the P-XRF methodology at this particular sampling target include the small effective sample size $(\approx 1$ $\mathrm{cm}^{3}$ ) and the small depth of analysis that might result in poor representativity, the bias on the estimated concentration values and the relatively high cost of the equipment. The first two limitations can be overcome by taking measurements on a finer grid so that variability can be observed at a smaller scale, and by excavation to extend analytical data to depth. Bias in comparison with laboratory results was corrected by accounting for the moisture content and applying a correction for surface roughness.

Further work is needed for the improvement of the performance of the method. The use of appropriate reference materials could be useful for the elimination of bias of the in situ measurements. However, it would be difficult to maintain materials simulating the soil in its natural state, i.e., containing sufficient moisture and with a matching grain size distribution. Comparisons of the concentration estimates with those from other established analytical methods are very important in the initial stages of development of an in situ analytical method. Finally, good planning of the survey is needed before using P$\mathrm{XRF}$ for in situ field-based analysis so as to be able to gain as much information as possible on estimates of both concentration values and uncertainties, to permit a realistic interpretation of the extent of contamination at the site.

The authors are very grateful to Peter Webb and Olwen Williams-Thorpe for field assistance with some of the P-XRF measurements. One of the authors (A.A.) is indebted to the Greek Scholarship Foundation for their support of this research.

\section{References}

1 Thompson, M., and Walsh, J. N., A Handbook of Inductively Coupled Plasma Spectrometry, Blackie, Glasgow, 1989.

2 Ramsey, M. H., Argyraki, A., and Thompson, M., Analyst, 1995, 120 1353.

3 Potts, P. J, Webb, P. C., Williams-Thorpe, O., and Kilworth, R., Analyst, 1995, 120, 1273.

4 Puls, R. W., Clark, D. A., Carlson, C., and Vardy, J., Ground Water Monit. Remed., 1994, 14, 111.

5 Kuharic, C. A., Cole, W. H., Singh, A. K., and Gonzales, D., EPA Report, EPA/600/R-93/073, US Environmental Protection Agency, Washington, DC, 1993.

6 Swift, R. P., Spectroscopy, 1995, 10, 31

7 Vincent, H. A., and Boyer, D. M., ASTM Special Technical Publication, STP 1226, American Society for Testing and Materials, Philadelphia, 1995, p. 215.

8 Kuharic, C. A., and Cole, W. H., Adv. X-Ray Anal., 1995, 38, 725.

9 Pyle, S. M., Nocerino, J. M., Deming, S. N., Palasota, J. A., Palasota, J. M., Miller, E. L., Hillman, D. C., Kuharic, C. A., Cole, W. H., Watson, M. A., and Nichols, K. D., Environ. Sci. Technol., 1995, 30, 204.

10 Argyraki, A., Ramsey, M. H., and Thompson, M., Analyst, 1995, 120, 2799.

11 Ramsey, M. H., Argyraki, A., and Thompson, M., Analyst, 1995, 120, 2309.

12 Maskall, J., and Thornton, I., Land Contam. Reclam., 1993, 1, 92.

13 Ramsey, M. H., Thompson, M., and Hale, M. J., Geochem. Explor., 1992, 44, 23.

14 Ripley, B. D., and Thompson, M., Analyst, 1987, 112, 377.

15 Potts, P. J., A Handbook of Silicate Rock Analysis, Blackie, Glasgow, 1987, p. 252.

16 Rose, A. W., Hawkes, H. E., and Webb, J. S., Geochemistry in Mineral Exploration, Academic Press, London, 1979. 